\title{
Adaptación e implementación de recursos didácticos para la enseñanza de ecuaciones de primer y segundo grado a niños con discapacidad visual en un aula inclusiva ${ }^{1}$
}

\author{
Adaptação e implementação de recursos pedagógicos para \\ equações de ensino de crianças primeiro e segundo grau \\ com deficiência visual em uma sala de aula inclusiva
}

\author{
Adaptation and implementation of teaching resources \\ for teaching equations of first and second grade children \\ with visual disabilities in an inclusive classroom
}

Recibido: mayo de 2013

Aprobado: agosto de 2013
Yenifer Paola Correa Hernández ${ }^{2}$

Erika Julieth Pulido Mahecha ${ }^{3}$

\section{Resumen}

Los procedimientos, gráficos, operaciones y procesos en las matemáticas hacen necesaria la implementación de recursos didácticos que permitan facilitar el aprendizaje de los contenidos de ella. Por esto son indispensables en la enseñanza de las matemáticas como instrumentos de apoyo que favorecen el proceso de matematización y representación de ideas matemáticas. Esto es una gran dificultad para el niño con discapacidad visual ya que en la educación matemática hacen falta materiales didácticos adaptados lo cuales mejoren el ritmo de trabajo y rendimiento a la hora de aprender haciendo uso de una Didáctica Especial de la Matemática para ciegos que permita una adecuación de materiales pedagógicos e instrumental de trabajo para esta población.

Palabras clave: Aula; recursos didácticos; materiales manipulativos; alumno; necesidades especiales; alumnos discapacitados; discapacidad visual; inclusión; matemáticas escolares; álgebra; ecuaciones.

\begin{abstract}
The procedures, graphics, operations and processes in mathematics require the implementation of teaching resources that will facilitate the learning of the contents of it. By this are essential in teaching mathematics as supporting tools that support the process of mathematization and representing mathematical ideas. This is a great difficulty for children with visual disabilities and mathematics education that are needed as adapted materials which improve the pace of work and performance when learning making
\end{abstract}

1 Artículo de Investigación.

2 Universidad Distrital Francisco José de Caldas. Bogotá, Colombia. Contacto: ypaitoch@hotmail.com

3 Universidad Distrital Francisco José de Caldas. Bogotá, Colombia. Contacto: julieth.erk@hotmail.com 
use of a Special Teaching Mathematics for the blind that allows adequacy of educational materials and instruments of work for this population.

Keywords: Classroom teaching resources, manipulatives, students, special needs students with disabilities, visual disabilities, inclusion, school mathematics, algebra equations.

\section{Resumo}

Os procedimentos, gráficos, operações e processos em matemática requer a aplicação de recursos pedagógicos que facilitem o aprendizado dos conteúdos do mesmo. Por isso são essenciais para o ensino da matemática como ferramentas de apoio que suportam o processo de matematização e representar idéias matemáticas. Esta é uma grande dificuldade para as crianças com deficiência visual e educação matemática que são necessários como materiais adaptados que melhoram o ritmo de trabalho e desempenho quando se aprende fazendo uso de um Ensino da Matemática especiais para cegos, que permite adequação dos materiais e instrumentos de trabalho de educação para essa população.

Palabras clave: Recursos em sala de aula de ensino, manipuláveis, estudantes, alunos com necessidades especiais, com deficiência, deficiência visual, inclusão escolar, matemática, equações de álgebra.

\section{Introducción}

En la educación es fundamental encontrar estrategias pedagógicas y didácticas que motiven a los estudiantes por aprender por sí mismos y con la guía del docente se construya de manera significativa su conocimiento y sean partícipes de él. Por esto, es de vital importancia el empleo de recursos didácticos adaptados a las necesidades educativas de cada alumno de tal manera que con la exploración y manipulación del mismo se construya de una manera real y tangible los conocimientos matemáticos.

Es obligación del Estado promover condiciones de igualdad realista y efectiva para todas las personas que posean limitaciones y es por esto que la ley General de Educación señala que en los establecimientos educativos se deben organizar los ritmos de aprendizaje dependiendo de las necesidades que presenten los estudiantes y ofrecer todas las herramientas de apoyo. De esta manera surge la importancia del empleo de material manipulativo tangible para el proceso de matematización y más para niños invidentes ya que según Rosich (1996) la vía más propicia para llevar a cabo esta matematización, es la manipulación de lo concreto porque los aspectos sensibles estimulan y facilitan el proceso de descubrimiento de lo matemático.

\section{Referentes Teórico - prácticos}

El pensamiento variacional aparece explícitamente en los lineamientos curriculares de matemáticas (MEN, 1994), donde se enfatiza la importancia de que los estudiantes adquieran destrezas que les permitan analizar, organizar y modelar matemáticamente situaciones y problemas tanto cotidianos como de otras ciencias. Por otra parte, algunas investigaciones muestran que la transición de la aritmética al algebra, es un proceso muy importante en el ámbito académico del estudiante, ya que cambia su panorama en el momento de enfrentarse a las expresiones algebraicas con variables, coeficientes, términos, factores e igualdades. Un modo de dar solución a estas ecuaciones es por medio de la transposición de términos, es decir, que deben realizar una serie de operaciones aritméticas donde se requiere el uso de las propiedades de las ecuaciones (Axiomas de igualdad) que contradicen lo realizado en sus primeros años escolares. Para el grupo 
Azarquiel (1993) un método de transposición de términos seria el "Deshacer" el cual consiste en aplicar al segundo término de la igualdad las operaciones inversas realizadas para hallar en el primer término. Este método está enfocado hacia el sentido operacional.

El material adaptado para niños videntes e invidentes se clasifica según Godino (2003) como material manipulativo tangible y se propone para la enseñanza-aprendizaje de las ecuaciones de primer grado "La Ficha tapada" y "La Balanza". Para el grupo Azarquiel (1993) el uso del material didáctico la "La Ficha tapada" define una ecuación como una identidad aritmética que tiene un número oculto que puede ser expresado por letras o espacios en blanco. El empleo de la "La Ficha tapada" permite un acercamiento al concepto intuitivo antes de su respectiva formalización a las ecuaciones algebraicas. Este material está conformado por fichas de números, letras y símbolos operatorios. "La Balanza según el grupo Arzaquiel (1993) permite trabajar el concepto de ecuación como una situación de equilibrio en ecuaciones generales como a y tiene el propósito de autocorrección ya que mediante su construcción física, el concepto de igualdad esta en relación con el equilibrio de las masas en ambos lados de la expresión. El material manipulativo tangible empleado para la enseñanza-aprendizaje de ecuaciones de segundo grado es el "Puzzle Algebraico", este permite representar geométricamente una expresión algebraica mediante una colección de figuras geométricas planas formada por cuadrados y rectángulos tales como se muestran en las siguientes figuras:

La representación geométrica de ecuaciones algebraicas de segundo grado se realiza término a término es decir:

Figura 1

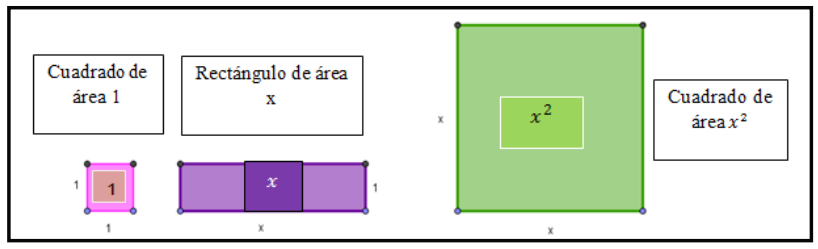

Fuente: Elaboración propia

- Termino cuadrático... se representa a través de la figura.
Figura 2

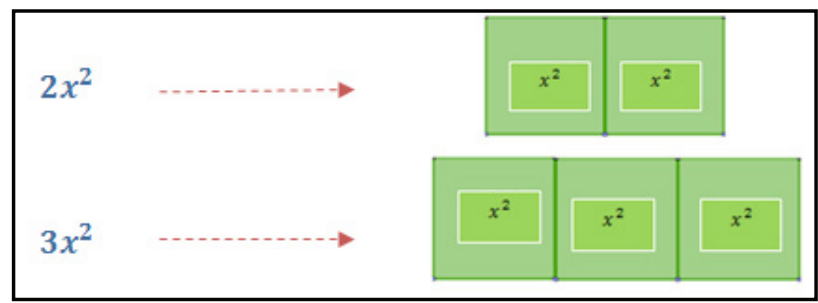

Fuente: Elaboración propia

- Término se representa a través de la figura de área.

Figura 3

$3 x$
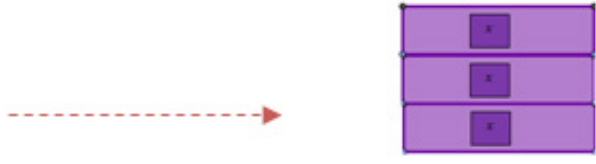

$4 x$

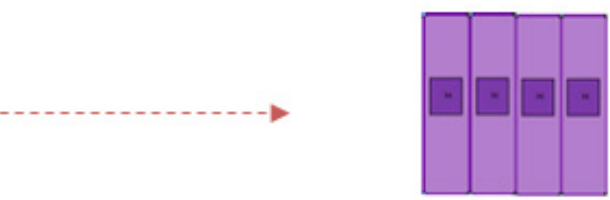

Fuente: Elaboración propia

- Termino independiente se representa a través de la figura de área 1

Figura 4

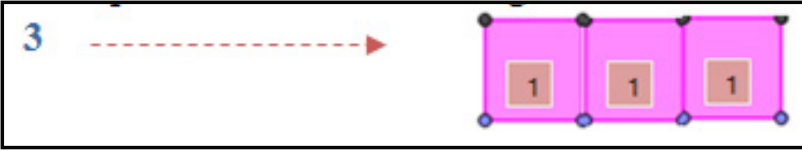

Fuente: Elaboración propia

Otro material en que se apoyó la experiencia fue el gráfico -textual que son las guías de trabajo y talleres donde cada una es diseñada para los estudiantes videntes e invidentes.

\section{Descripción general de la experiencia de aula}

El reto de lograr construir una secuencia didáctica enfocada a niños de octavo grado donde se destaca el uso de recursos didácticos manipulativos tangibles y gráfico textuales adaptados para niños videntes e invidentes resulto ser una experiencia enriquecedora e inolvidable para nosotros como estudiantes para profesor de matemáticas ya que 
era la primera vez que se trabajaba en un aula inclusiva con una estudiante con limitación visual. Fue un reto acomodar nuestro lenguaje instruccional y visual a un lenguaje más cuidadoso, claro y preciso para transmitir los conocimientos matemáticos. Esto nos llevó a dejar de lado las explicaciones en el tablero y reemplazarlas con la implementación de diversos recursos didácticos como: La ficha tapada, la balanza, el domino algebraico y guías en braille. Todos estos recursos adaptados para estudiantes videntes e invidentes que se caracterizaron por ser un material e instrumental de lectura el cual era diseñado en Braille con la ventaja de ser voluminoso; instrumental de escritura que se disponía de una regilla; instrumental de dibujo en relieve y a gran escala y material pedagógico auxiliar el cual era generador de situaciones didácticas (La ficha tapada, la balanza, domino algebraico).

Siempre fue importante la elaboración de un material comprensible que pudiera ser usado por todos los estudiantes en la clase ya que era importante que ellos estuvieran en un mismo nivel para avanzar en el desarrollo de las demás clases y tuvieran también una igualdad de condiciones. Además era de gran importancia que nuestra estudiante invidente tuviera un instructor de apoyo que le guiara en el momento de presentar dudas e inquietudes a medida que transcurría la clase. Fue así como esta experiencia nos doto de enriquecedores saberes como la escritura en braille y el uso de recursos didácticos además de la idea de pensar en un aula inclusiva, cosa que no fue tarea fácil ya que todos manejaban diferentes ritmos de aprendizaje pero que poco a poco se logró con la implementación de todos los materiales y las explicaciones cuidadosas por parte de las docentes.

\section{Logros y dificultades}

Con la adaptación e implementación de los recursos didácticos para la enseñanza de ecuaciones de primer y segundo grado se logró que los estudiantes en su mayoría reconocieran la estructura de una ecuación de primer y segundo grado ya que identifican los coeficientes y las variables de cada una de ellas; también se logró en los estudiantes una correcta transposición, simplificación y despeje de expresiones algebraicas de primer grado para hallar la respuesta, esto se evidencio con la ficha tapada. Con el recurso de la balanza se observó que los estudiantes identificaban el significado de igualdad como un equilibrio, y que para la resolución de estas era necesario hacer uso de propiedades en este caso el de quitar o agregar figuras. Por medio del puzle algebraico los estudiantes lograron identificar la estructura de una ecuación de segundo grado y algunos lograron la creación del cuadrilátero o rectángulo con el mismo. Las dificultades que se presentaron fueron que los estudiantes no identificaban la equivalencia de una expresión dada y tenían confusiones con las operaciones al usar. Confundían que al representar ecuaciones de segundo grado deben hacerse por medio de cuadrados o rectángulos y no por triángulos.

\section{Reflexión final}

Al implementar los recursos didácticos para la enseñanza- aprendizaje de los contenidos matemáticos hay que tener en cuenta que el uso del recurso por sí solo no garantiza un aprendizaje significativo por lo cual este debe ir acompañado de más estrategias metodológicas que conlleven al logro de los propósitos planteados en cada una de las actividades. El material manipulativo debe enfrentar a los estudiantes a situaciones problema que le permitan realizar una actividad reflexiva que pueda dar inicio o que pueda complementar el trabajo matemático realizado o a realizar en el aula. Por lo cual este material debe estar compuesto por una serie de reglas o parámetros que sean claros y de acuerdo al nivel de cada estudiante.

En el aula se tenía en cuenta la inclusión a los estudiantes ciegos, ya que ellos deben hacer parte de las clases con el mismo material para todos, por esto es necesario aprender y tener en cuenta que al planear actividades con algún recurso didáctico este debe estar sujeto a cualquier tipo de adaptación para que 
sea accesible para todo tipo de estudiante. Y que dicho recurso sea manipulativo para ellos y cumpla el propósito de enseñar algo.

\section{Referencias}

Ministerio de Educación Nacional (1994). Lineamientos curriculares para el área de matemáticas. Bogotá D.C.

GODINO. J (2003). Uso de material tangible y gráfico-textual en el estudio de las matemáticas: superando algunas suposiciones ingenuas.
Vasco, Carlos.(S.F.). Algunas reflexiones sobre la pedagogía y la didáctica. Bogotá D.C, COLCIENCIAS.

Dulciani, B. (1976). Algebra Moderna, Estructura y Método.

Grupo Azarquiel. (1993). Ideas y Actividades Para Enseñar Algebra. Bogotá D.C, Síntesis.

Rosich, N. (1996). Matemáticas y Deficiencia Sensorial. Bogotá D.C, Síntesis. 